\title{
Genome sequence of Shigella flexneri strain SP1, a diarrheal isolate that encodes an extended-spectrum $\beta$-lactamase (ESBL)
}

Ping Shen ${ }^{1+}$, Jianzhong Fan ${ }^{2+}$, Lihua Guo ${ }^{1}$, Jiahua $\mathrm{Li}^{3}$, Ang $\mathrm{Li}^{1}$, Jing Zhang ${ }^{1}$, Chaoqun Ying ${ }^{1}$, Jinru Ji ${ }^{1}$, Hao Xu', Beiwen Zheng ${ }^{1 *}$ and Yonghong Xiao ${ }^{1}$

\begin{abstract}
Background: Shigellosis is the most common cause of gastrointestinal infections in developing countries. In China, the species most frequently responsible for shigellosis is Shigella flexneri. S. flexneri remains largely unexplored from a genomic standpoint and is still described using a vocabulary based on biochemical and serological properties. Moreover, increasing numbers of ESBL-producing Shigella strains have been isolated from clinical samples. Despite this, only a few cases of ESBL-producing Shigella have been described in China. Therefore, a better understanding of ESBL-producing Shigella from a genomic standpoint is required. In this study, a S. flexneri type 1a isolate SP1 harboring bla $\mathrm{a}_{\text {CTX-M-14, }}$ which was recovered from the patient with diarrhea, was subjected to whole genome sequencing.
\end{abstract}

Results: The draft genome assembly of S. flexneri strain SP1 consisted of 4,592,345 bp with a G+C content of 50.46\%. RAST analysis revealed the genome contained 4798 coding sequences (CDSs) and 100 RNA-encoding genes. We detected one incomplete prophage and six candidate CRISPR loci in the genome. In vitro antimicrobial susceptibility testing demonstrated that strain SP1 is resistant to ampicillin, amoxicillin/clavulanic acid, cefazolin, ceftriaxone and trimethoprim. In silico analysis detected genes mediating resistance to aminoglycosides, $\beta$-lactams, phenicol, tetracycline, sulphonamides, and trimethoprim. The bla $a_{\text {CTX-M-14 }}$ gene was located on an IncFII2 plasmid. A series of virulence factors were identified in the genome.

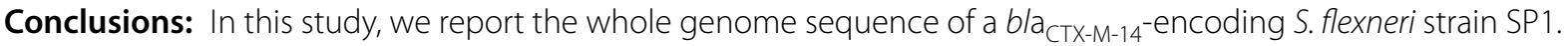
Dozens of resistance determinants were detected in the genome and may be responsible for the multidrug-resistance of this strain, although further confirmation studies are warranted. Numerous virulence factors identified in the strain suggest that isolate SP1 is potential pathogenic. The availability of the genome sequence and comparative analysis with other $\mathrm{S}$. flexneri strains provides the basis to further address the evolution of drug resistance mechanisms and pathogenicity in S. flexneri.

Keywords: Shigella flexneri, Extended-spectrum $\beta$-lactamase, IncFII2, Comparative genomic analysis

\section{Background}

Shigella species are a major causative cause of gastrointestinal infections throughout the world, especially

\footnotetext{
*Correspondence: zhengbw@zju.edu.cn

†Ping Shen and Jianzhong Fan contributed equally to this article

${ }^{1}$ Collaborative Innovation Center for Diagnosis and Treatment

of Infectious Diseases, State Key Laboratory for Diagnosis and Treatment

of Infectious Diseases, The First Affiliated Hospital, School of Medicine,

Zhejiang University, Hangzhou 310003, China

Full list of author information is available at the end of the article
}

in developing countries [1]. Globally, there are approximately 164.7 million cases per annum, of which 1.1 million people are estimated to die from Shigella infections [2]. Based on biochemical and serological properties, the genus Shigella comprises four serogroups: Shigella dysenteriae, Shigella flexneri, Shigella boydii and Shigella sonnei [2]. S. flexneri is endemic in many developing countries and causes more deaths than any other Shigella serotypes [3]. In China, shigellosis is the most common gastrointestinal infections [4] and most frequently 
isolated species responsible for shigellosis in mainland China is S. flexneri [5].

Antibiotic treatment is usually recommended for shigellosis as it reduces the duration and severity of symptoms, reduces the excretion of organisms and prevents potentially lethal complications [6]. However, the emergence of extendedspectrum $\beta$-lactamase (ESBL)-producing $S$. flexneri is a major public health problem in China, as these strains are associated with critical infections [7]. Most of the CTX-M, SHV, and TEM-type ESBLs genes are located on conjugative plasmids [4]. Moreover, co-existence with other antimicrobial resistance genes is frequently observed in ESBL-producers [8], which makes the choice of effective treatment extremely limited. Increasing instances of ESBL-producing Shigella strains isolated from Asia have been reported [1, 2]. So far, only a few cases of ESBL-producing Shigella have been described in China [4]. Therefore, a better understanding of ESBL-producing Shigella using a genomics approach is required.

The first genome sequence of $S$. flexneri was reported by Jin et al. in 2002 [9]. So far, more than 145 S. flexneri strains have been sequenced and analyzed [10]. To extend our understanding of the resistance mechanisms and pathogenesis of ESBL-producing S. flexneri, we performed sequencing and genomic analysis of the ESBL-harbouring S. flexneri SP1. Comparative genomics analysis of S. flexneri SP1 with other S. flexneri genomes may improve our understanding of the antibiotic resistance and virulence factors present in Shigella.

\section{Methods}

\section{Strain information}

Shigella flexneri 1a isolate SP1 was isolated from the stool sample of a 76-year-old female diarrhea patient. Standard biochemical tests were performed using the Vitek II system (BioMerieux, France) and species-specific 16S rRNA sequencing was used to confirm the identity of isolate SP1. Serotyping was performed with specific antiserum (Denka-Seiken). Genomic DNA of SP1 was extracted from a single colony of the pure bacterial culture. Possible contamination with other DNA and misassemblies were assessed by performing a BLAST search against the non-redundant database as described previously [11]. The whole genome of SP1 is in the expected size range for a Shigella genome and the coverage of the reads was consistent throughout the genome. The assembled draft genome sequence of SP1 was further verified by comparative analysis with the published complete genome sequences of S. flexneri strains.

\section{Antimicrobial susceptibility testing}

Susceptibility testing for ampicillin, amoxicillin/clavulanic acid, amikacin, aztreonam, piperacillin/tazobactam, cefazolin, cefoxitin, ciprofloxacin, ceftriaxone, cefepime, ertapenem, imipenem, gentamycin, tobramycin, levofloxacin, tigecycline, nitrofurantoin, and trimethoprim were performed by the microbroth dilution method and interpreted according to the CLSI guidelines [12]. Escherichia coli strain ATCC 25922 was used as the control strain for susceptibility studies. Late log phase cells were harvested, and genomic DNA was extracted from the pure culture using the DNeasy Blood \& Tissue kit (Qiagen, Germany) according to the manufacturer's instructions. For the purpose of bacterial identification, we amplified the $16 \mathrm{~S}$ rRNA gene with a 16S rRNA universal primer set and the PCR product was sequenced. Ethical approval was granted by the Ethics Committee of the First Affiliated Hospital of Zhejiang University.

\section{Genome sequencing and assembly}

The extracted DNA was visualized by agarose gel electrophoresis and quantitated by Qubit 2.0. Whole-genome sequencing was performed on the Illumina HiSeq 4000PE150 platform. DNA was tailed, ligated to paired-end adaptors and PCR amplified with a $500 \mathrm{bp}$ insert size and a mate-pair libraries with an insert size of $5 \mathrm{~kb}$ were used for library construction at the Beijing Novogene Bioinformatics Technology Co., Ltd. Illumina PCR adapter reads and low quality reads from the paired-end and mate-pair library were filtered by the quality control step using Novogene pipeline. All high quality paired reads were assembled using Velvet 1.2.10 [13] into a number of scaffolds. The filtered reads were then passed handled by the next step of the gap-closing.

\section{Genome annotation}

Genome annotation included the prediction of coding genes, transfer RNAs, ribosomal RNA, prophage, and clustered regularly interspaced short palindromic repeat sequences (CRISPR). Open reading frames (ORFs) were identified and classified using the Rapid Annotation using Subsystem Technology (RAST) server [14]. Protein classification into functional groups was performed using the Clusters of Orthologous Groups of proteins (COGs) [15]. Transfer RNAs and ribosomal RNA genes rRNAs were detected by tRNAscan-SE [16] and RNAmmer 1.2 software [17], respectively. PHASTER [18] was used to identify prophage and putative phage-like elements and CRISPRFinder [19] was used to identify CRISPR sites. The plasmid replicon was predicted by the PlasmidFinder Tool [20]. ISfinder [21] was employed to search for IS sequences in the genome, with an $\mathrm{e}$-value of $1 \mathrm{E}-3$. plasmidSPAdes was used to produce plasmid sequences from the WGS data [22].

\section{Antibiotic resistance genes prediction and virulence factors analysis}

Antibiotic resistance genes were annotated using the comprehensive antibiotic resistance database (CARD) 
[23] and Resfinder [24] with default parameters. We further verified all putative antibiotic resistance genes (ARGs) through a BLAST search with cut-off e-value of $1 \mathrm{E}-0.5$. Virulence factors were predicted by using BLAST to search against the VFDB database [25] with an e-value threshold of 1E-5 and also with VirulenceFinder 1.5 [26].

\section{Plasmid characterization}

PlasmidFinder 1.3 was used for identify the incompatibility group of the plasmid present in S. flexneri SP1 [20]. The plasmid sequence of $b l a_{\mathrm{CTX}-\mathrm{M}-14}$-harboring plasmid from isolate SP1 (named pSP1) was assembled with plasmidSPAdes [22]. Assignment of the plasmid to an incompatibility (Inc) group was performed by multiplex PCR. PCRs were performed as described previously [27].

\section{Phylogenetic analysis and comparative genomic analysis}

Comparative genomic analysis was performed by orthology identification method as previously described [11, $28]$. Genome sequences of the following representative $S$. flexneri strains were downloaded from the NCBI genome database: S. flexneri 2a strain 981 (CP012137), S. flexneri S7737 (AMJY00000000), S. flexneri 5a strain M90T (CM001474), S. flexneri CDC 796.83 (AERO00000000), S. flexneri 4343.70 (AFHC00000000), S. flexneri NCTC1 (LM651928), S. flexneri 2a strain 301 (AE005674), S. flexneri G1663 (CP007037), S. flexneri Shi06HN006 (CP004057), S. flexneri 2003036 (CP004056), S. flexneri str 4S BJ10610 (JMRK00000000), S. flexneri 4c strain 1205 (CP012140), S. flexneri 2002017 (CP001383) and S. flexneri 1a strain 0228 (CP012735). Phylogenetic reconstruction and analysis was performed wih the phangorn package, written in the $\mathrm{R}$ language [29]. VennDiagram [30] was used to generate the Venn plots of S. flexneri SP1, S. flexneri str 4S BJ10610, S. flexneri 4c strain 1205 (CP012140), S. flexneri 2002017, and S. flexneri 1a strain 0228.

\section{Results and discussion General features}

We performed whole genome sequencing using the Illumina HiSeq 4000 system with $2 \times 150$ bp paired-end reads. After quality control, we assembled the $1095 \mathrm{M} \mathrm{bp}$ filtered reads into contigs. The assembled genome of $S$. flexneri SP1 revealed a genome size of 4,592,345 bp with a G+C content of $50.46 \%$. The largest contig consisted of $137,097 \mathrm{bp}$ and the length of N50 contig was 33,394 bp. These scaffolds contain 4798 coding sequences (CDSs), and 100 RNA-encoding genes. The properties and the statistics of the genome are summarized in Additional file 1: Table S1. The resulting genomic size of strain SP1 was similar to previous studies within the range of 4.1-4.8 M bp [31, 32]. Similarly, CDSs numbers were close to the previous publication [31]. Gene functions were predicted using RAST and COG analysis. RAST server based annotation of the whole genome describes the distribution of subsystems in strain SP1 (Fig. 1a). Proteins responsible for carbohydrates (693 ORFs), amino acids and derivatives (384 ORFs), and cofactors, vitamins, prosthetic groups, pigments (292 ORFs) were abundant among the subsystem categories. The distribution of COGs is illustrated in Fig. 1b. The most abundant COG categories were R (general function prediction only), S (function unknown), $\mathrm{E}$ (amino acid transport and metabolism), G (carbohydrate transport and metabolism) and $\mathrm{K}$ (transcription). Furthermore, one incomplete prophage region was identified in the genome of SP1. It is a Salmonella ST64B-like phage (Acc-No. NC_004313) of $14.1 \mathrm{~kb}$ in length and a G+C content of $50.81 \%$. Additionally, six questionable CRISPR loci were detected by CRISPERfinder.

\section{Antimicrobial susceptibility profiles and antibiotic resistance genes}

The in vitro antimicrobial susceptibility testing demonstrated that the strain SP1 was resistant to ampicillin, amoxicillin/clavulanic acid, cefazolin, ceftriaxone and trimethoprim, but susceptible to piperacillin/tazobactam, cefoxitin, cefepime, aztreonam, imipenem, amikacin, gentamicin, tobramycin, ciprofloxacin, levofloxacin, tigecycline and nitrofurantoin (Additional file 2: Table S2). We then screened the antibiotic resistance genes (ARGs) in the genome to further explore the genetic basis of multidrug resistance in this strain (Additional file 3: Table S3). In silico analysis revealed the presence of some putative ARGs for different drug classes. We detected genes mediating resistance to aminoglycosides (aadA24, strA and $\operatorname{str} B), \beta$-lactams $\left(b l a_{\mathrm{CTX}-\mathrm{M}-14}\right.$ and $\left.b l a_{\mathrm{OXA}-1}\right)$, phenicol (catA1), tetracycline (tetD), sulphonamides (sul2), and trimethoprim (dfrA1). CTX-M-14 was the most frequent ESBL variant detected in Shigella isolates in China, followed by CTX-M-15 [4, 33]. Hitherto, only a few studies have reported the presence of ESBLs in S. flexneri [34]. Interestingly, all of the $b l a_{\mathrm{CTX}-\mathrm{M}-14-}$ harbouring $S$. flexneri strains were isolated from China $[4,7,34,35]$. A previous study reported a high prevalence of extended-spectrum cephalosporin resistance Shigella mediated mainly by $b l a_{\text {CTX-M }}$ (mainly $b l a_{\text {CTX-M-14 }}, 14.1 \%$ ) in Hangzhou City, Zhejiang Province, China [35]. Our study further indicates the existence of $b l a_{\text {CTX-M-14- }}$ harbouring $S$. flexneri clone may responsible for these Shigella infections in this area. In addition, the insertion sequence (IS) elements were frequently detected in upstream of $b l a_{\text {CTX-M }}$ genes [36]. ISfinder was thus employed to scan the $b$ la $_{\text {CTX-M-14 }}$ flanking sequences in a range of $6-\mathrm{kb}$ for IS sequences and junction associated proteins. ISEcp 1 and IS903B were found upstream of $b l \mathrm{a}_{\mathrm{CTX}-\mathrm{M}-14}$. ISEcp1 belongs to 


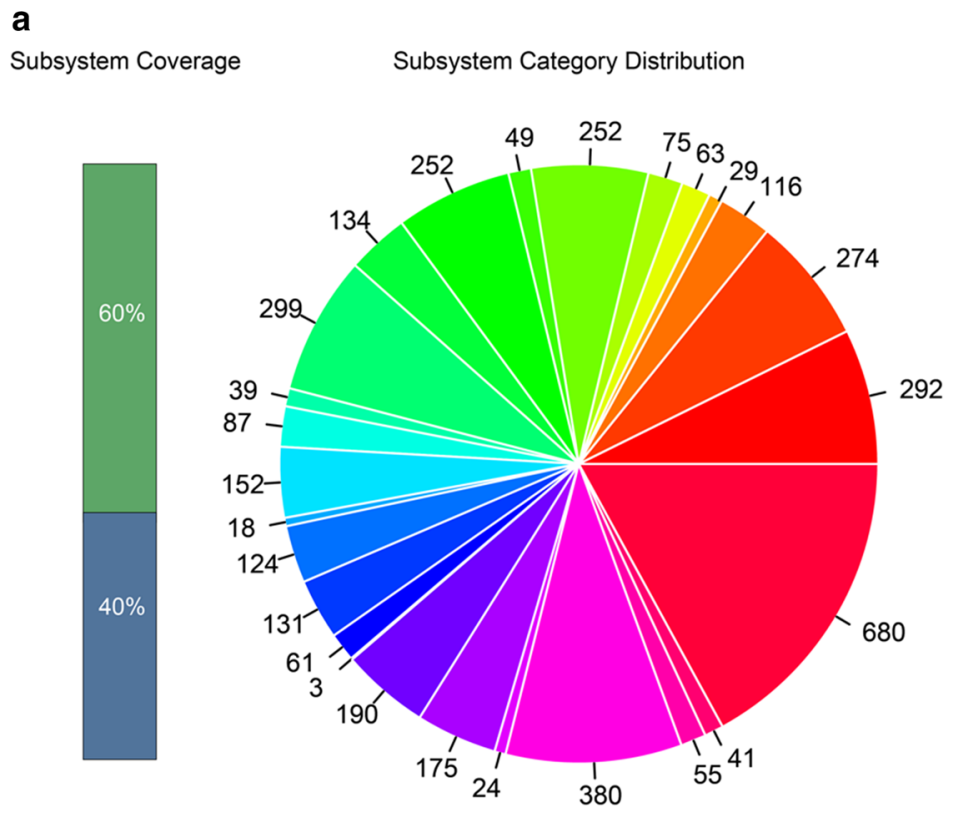

Subsystem Feature

b

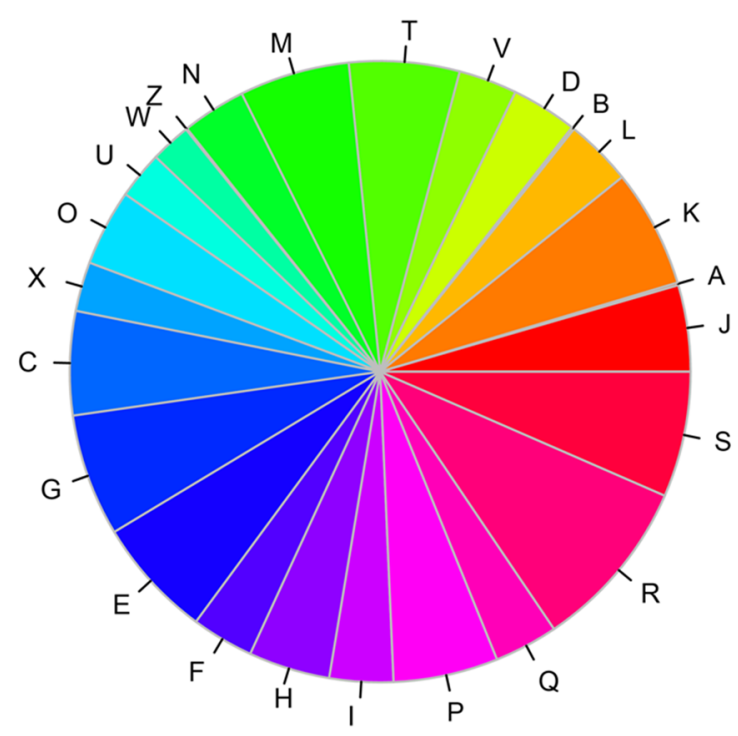

1-Cofactors, Vitamins, Prosthetic Groups, Pigments(292)

2-Cell Wall and Capsule(274)

$\square$ 3-Virulence, Disease and Defense(116)

$\square$ 4-Potassium metabolism(29)

$\square$ 5-Photosynthesis(0)

$\square$ 6-Miscellaneous(63)

$\square$ 7-Phages, Prophages, Transposable elements, Plasmids(75)

$\square$ 8-Membrane Transport(252)

$\square$ 9-Iron acquisition and metabolism(49)

ㅁ 10-RNA Metabolism(252)

$\square$ 11-Nucleosides and Nucleotides(134)

$\square$ 12-Protein Metabolism(299)

ㅁ 13-Cell Division and Cell Cycle(39)

$\square$ 14-Motility and Chemotaxis(87)

$\square$ 15-Regulation and Cell signaling(152)

16-Secondary Metabolism(18)

17-DNA Metabolism(124)

- 18-Fatty Acids, Lipids, and Isoprenoids(131)

- 19-Nitrogen Metabolism(61)

20-Dormancy and Sporulation(3)

21-Respiration(190)

- 22-Stress Response(175)

$\square$ 23-Metabolism of Aromatic Compounds(24)

- 24-Amino Acids and Derivatives(380)

25-Sulfur Metabolism(55)

26-Phosphorus Metabolism(41)

27-Carbohydrates (680)

- J:Translation, ribosomal structure and biogenesis

- A:RNA processing and modification

E:Transcription

L:Replication, recombination and repair

B:Chromatin structure and dynamics

- D:Cell cycle control, cell division, chromosome partitioning

- V:Defense mechanisms

- T:Signal transduction mechanisms

- M:Cell wall/membrane/envelope biogenesis

- N:Cell motility

Z:Cytoskeleton

W:Extracellular structures

- U:Intracellular trafficking, secretion, and vesicular transport

- O:Posttranslational modification, protein turnover, chaperones

- X:Mobilome: prophages, transposons

- C:Energy production and conversion

- G:Carbohydrate transport and metabolism

- E:Amino acid transport and metabolism

- F:Nucleotide transport and metabolism

- $\mathrm{H}:$ Coenzyme transport and metabolism

- I:Lipid transport and metabolism

- P:Inorganic ion transport and metabolism

Q:Secondary metabolites biosynthesis, transport and catabolism

- R:General function prediction only

- S:Function unknown

Fig. 1 Analysis of annotated genes in S. flexneri strain SP1 based on the SEED and COG databases. a The green bar represents the percentage of proteins that were annotated by RAST server, while the blue bar indicated the proteins not annotated. The pie chart demonstrates the abundance of each subsystem category and the count of each subsystem feature is shown. b Distribution of COGs. Each bar indicates the number of annotated genes based on the COG database

the IS1380 family, which may enhance the expression of $b l a_{\mathrm{CTX}-\mathrm{M}-14 /-18}, b l a_{\mathrm{CTX}-\mathrm{M}-17}$ and $b l a_{\mathrm{CTX}-\mathrm{M}-19} \beta$-lactamase genes [37].

\section{Genetic context of $b / a_{\text {CTX-M-14 }}$ gene}

PlasmidFinder and plasmidSPAdes were used to detect the potential plasmids in the whole genome sequence. In silico analysis revealed that $b l a_{\mathrm{CTX}-\mathrm{M}-14}$ was located on an IncFII2 plasmid. To further explore the genetic environment of the $b l a_{\mathrm{CTX}-\mathrm{M}-14}$ gene in isolate SP1, using the bla $_{\text {CTX-M-14 }}$ carrying contig as a query against the $\mathrm{nr} /$ nt database revealed sequence homology to the $\sim 74 \mathrm{~kb}$ annotated $b l a_{\text {CTX-M-14-positive IncFII2 plasmid pAC2901 }}$ (GenBank: KU987452) from Citrobacter freundii strain AC2901 (Fig. 2). Multiple sequence alignments demonstrated that DNA sequences between pSP1 and pAC2901 
share $>99 \%$ identity. Successful dissemination of $b l a_{\text {CTX- }}$ M-14 among Enterobacteriaceae isolates from humans, animals and the environment has mainly been associated with IncK, IncF and IncI1 plasmids [38], and there has been only 1 report of a $b l a_{\text {CTX-M-14 }}$ located on an IncFII2 plasmid in the English literature [39]. Here we report for the first time an IncFII2 $b l \mathrm{a}_{\mathrm{CTX}-\mathrm{M}-14}$-encoding plasmid in the genus Shigella.

\section{Pathogenesis and virulence factors}

Shigella flexneri remains a public health concern throughout the world and its pathogenesis should be further investigated. We performed a BLASTP search against the VRDB database and found several known virulence factors. These virulence factors include Shigella extracellular protein A (sepA), glutamate decarboxylase ( $\mathrm{gadA}$ ), invasion plasmid antigen (ipaH9.8), long polar fimbriae (lpfA), hexosyltransferase homolog (capU), invasion protein Shigella flexneri (ipaD), serine protease autotransporters of Enterobacteriaceae (pic), VirF transcriptional activator (virF), and Shigella IgA-like protease homologue (sigA). The ability to withstand an acid-challenge of $\mathrm{pH} 2.5$ by $S$. flexneri is a necessary virulence trait, which requires acid-induction of a functional GdaA in the stationary-growth phase [40]. IpaH9.8 is a member of the IpaH family of Shigella, which are encoded on the $220 \mathrm{~kb}$ virulence plasmid or chromosome and has been shown to be secreted into the host cell where it is targeted to the nucleus [41]. Moreover, LpfA has been linked to virulence in enterohemorrhagic E. coli [42]. Earlier reports indicated the SigA is cytopathic for HEp-2 cells and contributes to the intestinal fluid accumulation associated with $S$. flexneri infections [43]. Translocation of effector proteins into host cells and the surrounding space is a common strategy used by $S$. flexneri to target signaling pathways in the host cell [44], and IpaD and VirF are required to facilitate bacterial invasion of host cells [45]. More importantly, Pic is secreted by pathogenic Gram-negative bacteria through the autotransporter pathway and targets a broad range of human leukocyte adhesion proteins, which represent unique immune-modulating bacterial virulence factors [46]. These data suggest that isolate SP1 is potential pathogenic, which is consistent with the isolation of SP1 from a diarrhea patient.

\section{Comparative analysis with other S. flexneri strains}

Based on genomes downloaded from the NCBI database, phylogenetic analysis was performed and the resulting tree topology was assessed to identify genetic relatedness between 14 S. flexneri isolates and strain SP1 (Fig. 3a). This revealed that SP1 is most closely related to $S$. flexneri str $4 \mathrm{~S}$ BJ10610, which was also isolated from a severe diarrhea patient and was resistant to multiple drugs [47]. Other $S$. flexneri strains are also has highly similar, except for S. flexneri CDC 796.83, suggesting that S. flexneri strains show high similarity between different species. A previous study has highlighted that $S$. flexneri has a stable core genome that is equipped with a repertoire of virulence determinants that have enabled it to colonize, and persist, in multiple locations for hundreds of years [48]. A functional genomic comparison was performed between strain SP1 and its four most closely related neighbors: S. flexneri str 4S BJ10610 (JMRK00000000), S. flexneri 2002017 (CP001383), S. flexneri 4c str 1205 (CP012140), and S. flexneri 1a str 0228 (CP012735). The Venn diagram indicates the presence of 4449 core conserved genes present in the pan-genome of the analyzed S. flexneri isolates (Fig. 3b). Interestingly, a total of 178 strain-specific genes were identified in strain SP1.

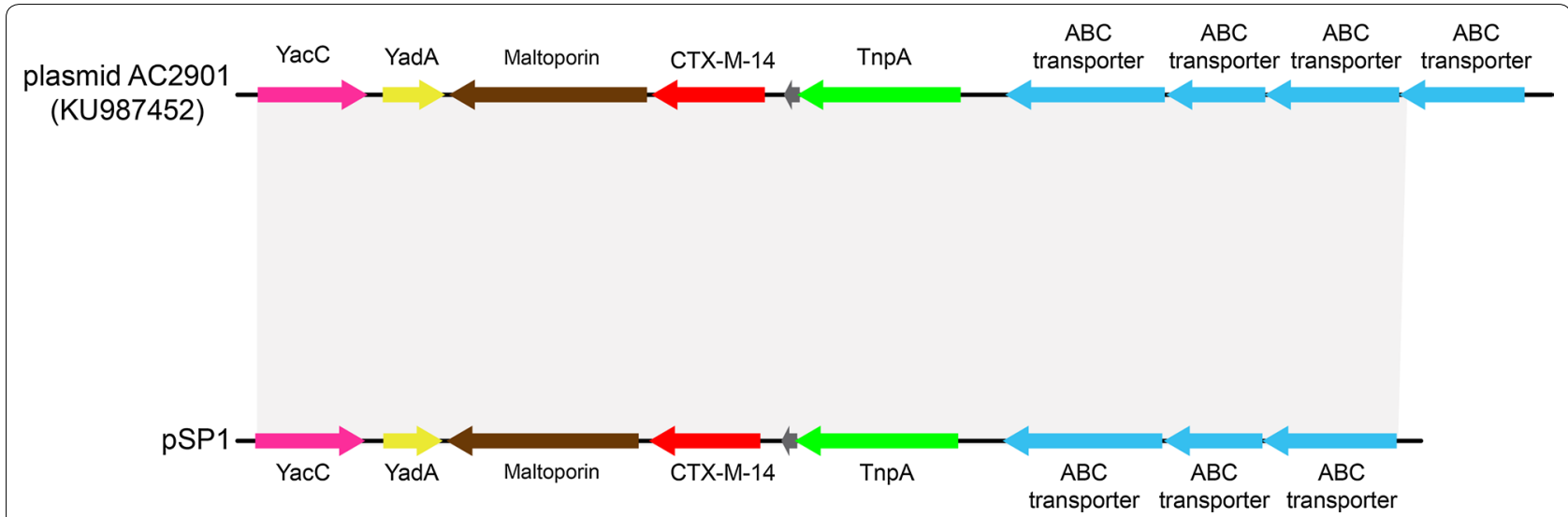

Fig. 2 Genetic organization of scaffolds (portions of genome sequences reconstructed from the whole-genome sequence) containing bla ${ }_{C T X-M-14}$ harbored by plasmid pSP1 and structural comparison with plasmid pAC2901. Arrows indicate positions and direction of transcription of genes. Regions with >99\% homology are shown in gray. Information in parentheses after isolates represents the GenBank accession number 
a

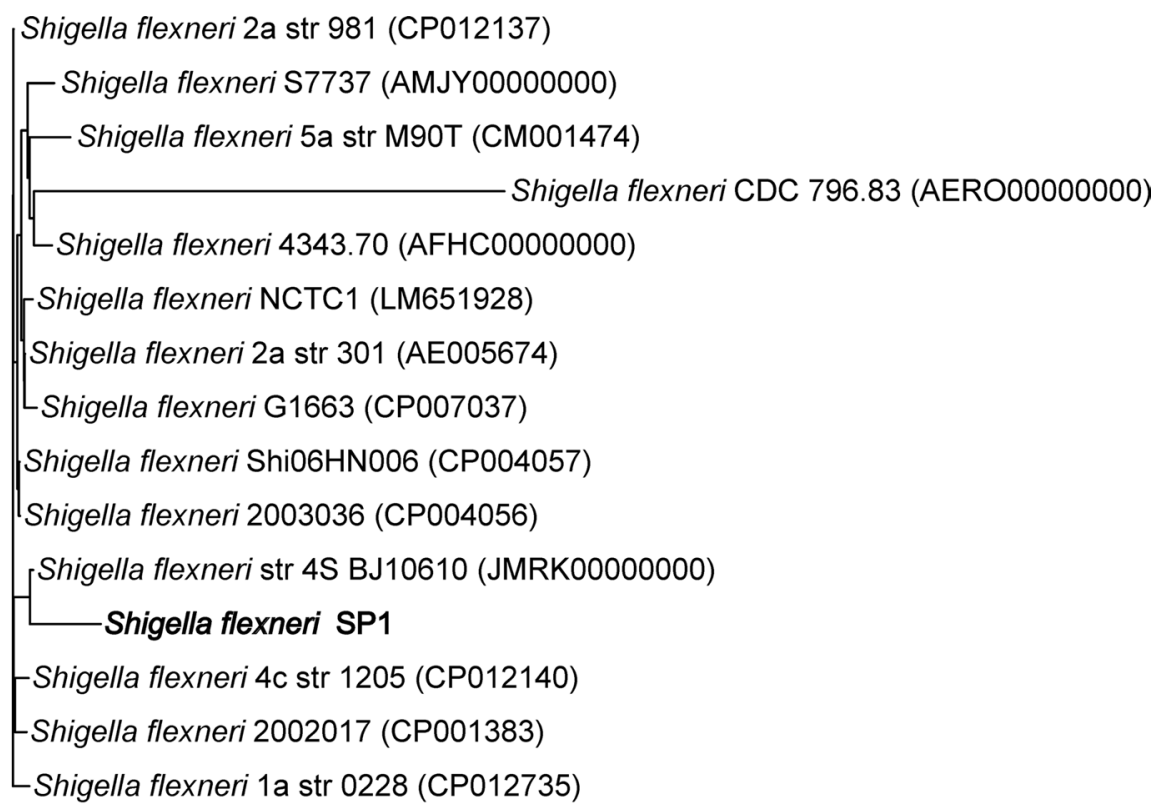

b

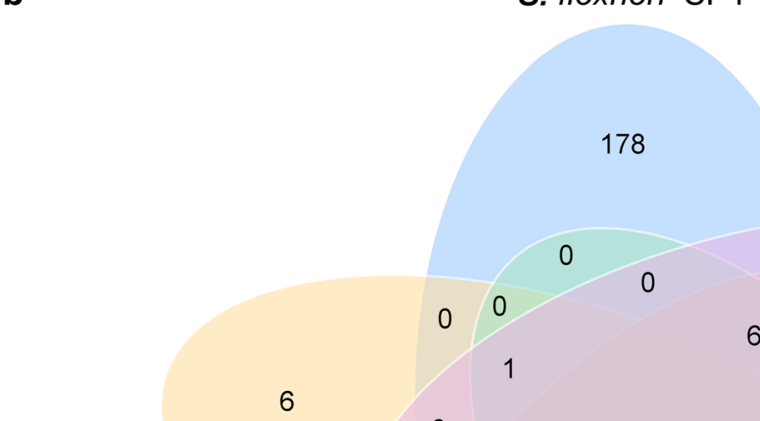

S. flexneri 4S BJ10610
0

0

\section{S. flexneri SP1}

\section{0}

\section{8}

30

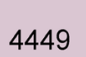

4449

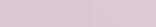

0

0

170

01

0

0

0

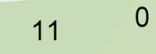

0

S. flexneri 1a str 0228 $\begin{array}{lll}0 & 0 & 16\end{array}$

28

\section{S. flexneri}

4c str 1205

\section{S. flexneri} 2002017

Fig. 3 a Phylogenetic tree of S. flexneri strain SP1 with 14 other S. flexneri isolates. The tree was constructed based on based on alignments of orthologous genes. b Venn diagrams showing the orthologous groups in the five most closely related S. flexneri genomes. Numbers inside the Venn diagrams indicated the number of genes found to be shared among the indicated genomes

\section{Conclusions}

As more S. flexneri genomes have been sequenced in recent years, comparative genomic studies have progressed rapidly. So far, whole genome studies of $S$. flexneri have exclusively focused on the historical global spread and recent local persistence among these isolates. This work is the first description of the draft genome of

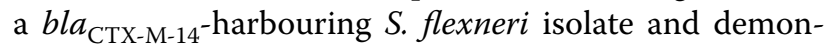
strates the compares the genome of strain SP1 to other $S$. flexneri isolates. However, the data presented here is a 
preliminary report on the virulence profile and antibiotic resistance of S. flexneri strain SP1. Future studies involving more ESBL-encoding $S$. flexneri isolates from China are urgently needed to study the dynamics of the dissemination of ESBL genes, especially the complete sequence of plasmids carrying these ESBL genes.

\section{Additional files}

Additional file 1: Table S1. Summary of S. flexneri strain SP1 genome.

Additional file 2: Table S2. Antimicrobial susceptibility profile of $S$. flexneri SP1 recovered from a patient with diarrhea.

Additional file 3: Table S3. Antibiotic resistance genes predicted in the S. flexneri strain SP1 genome.

\section{Authors' contributions}

$B Z, P S$ and JF were involved in the overall experimental design. PS, JF, LG, JL, $H X, J J$ and CY performed microbiology and molecular biology experiments. $B Z, L G$ and AL generated and analyzed the sequencing data. BZ participated in all discussions of data analysis and wrote the manuscript. All authors read and approved the final manuscript.

\section{Author details}

${ }^{1}$ Collaborative Innovation Center for Diagnosis and Treatment of Infectious Diseases, State Key Laboratory for Diagnosis and Treatment of Infectious Diseases, The First Affiliated Hospital, School of Medicine, Zhejiang University, Hangzhou 310003, China. ${ }^{2}$ Department of Clinical Laboratory, Hangzhou First People's Hospital, Hangzhou 310006, China. ${ }^{3}$ Department of Hospital Infection Control, Zhucheng People's Hospital, Zhucheng 252300, China.

\section{Acknowledgements}

Not applicable.

\section{Competing interests}

The authors declare that they have no competing interests.

\section{Availability of data and materials}

Whole Genome Shotgun project of Shigella flexneri strain SP1 has been deposited at DDBJ/EMBL/GenBank under the accession MTPK00000000. The version described in this paper is version MTPK00000000.

\section{Ethics approval and consent to participate}

This research was approved by the Research Ethics Committee of the First Affiliated Hospital, School of Medicine, Zhejiang University.

\section{Funding}

This work was supported by the National Natural Science Foundation of China (Nos. 81301460 and 81301461); National Key Research and Development Program of China (No. 2016YFD0501105).

\section{Publisher's Note}

Springer Nature remains neutral with regard to jurisdictional claims in published maps and institutional affiliations.

Received: 9 February 2017 Accepted: 4 May 2017 Published online: 12 May 2017

\section{References}

1. Taneja N, Mewara A, Kumar A, Verma G, Sharma M. Cephalosporinresistant Shigella flexneri over 9 years (2001-2009) in India. J Antimicrob Chemother. 2012;67:1347-53.
2. Tajbakhsh M, Garcia Migura L, Rahbar M, Svendsen CA, Mohammadzadeh M, Zali MR, Aarestrup FM, Hendriksen RS. Antimicrobial-resistant Shigella infections from Iran: an overlooked problem? J Antimicrob Chemother. 2012;67:1128-33.

3. Bennish ML, Wojtyniak BJ. Mortality due to shigellosis: community and hospital data. Rev Infect Dis. 1991;13(Suppl 4):S245-51.

4. Zhang R, Zhou HW, Cai JC, Zhang J, Chen GX, Nasu M, Xie XY. Serotypes and extended-spectrum beta-lactamase types of clinical isolates of Shigella spp. from the Zhejiang province of China. Diagn Microbiol Infect Dis. 2011;69:98-104.

5. Yang C, Li P, Zhang X, Ma Q, Cui X, Li H, Liu H, Wang J, Xie J, Wu F, et al. Molecular characterization and analysis of high-level multidrugresistance of Shigella flexneri serotype $4 \mathrm{~s}$ strains from China. Sci Rep. 2016;6:29124

6. Xu Y, Zhuang L, Kang H, Ma P, Xu T, Pan S, Gu B. Prevalence, resistance patterns, and characterization of integrons of Shigella flexneri isolated from Jiangsu Province in China, 2001-2011. Eur J Clin Microbiol Infect Dis. 2016:35:1347-53.

7. Zhang W, Luo Y, Li J, Lin L, Ma Y, Hu C, Jin S, Ran L, Cui S. Wide dissemination of multidrug-resistant Shigella isolates in China. J Antimicrob Chemother. 2011;66:2527-35.

8. Zheng B, Dong H, Xu H, Lv J, Zhang J, Jiang X, Du Y, Xiao Y, Li L. Coexistence of MCR-1 and NDM-1 in clinical Escherichia coli isolates. Clin Infect Dis. 2016;63:1393-5.

9. Jin Q, Yuan Z, Xu J, Wang Y, Shen Y, Lu W, Wang J, Liu H, Yang J, Yang F, et al. Genome sequence of Shigella flexneri 2a: insights into pathogenicity through comparison with genomes of Escherichia coli K12 and O157. Nucleic Acids Res. 2002;30:4432-41.

10. Ashton PM, Baker KS, Gentle A, Wooldridge DJ, Thomson NR, Dallman $\mathrm{TJ}$, Jenkins C. Draft genome sequences of the type strains of Shigella flexneri held at Public Health England: comparison of classical phenotypic and novel molecular assays with whole genome sequence. Gut Pathog. 2014;6:7.

11. Wang T, Jiang X, Feng C, Li A, Dong H, Wu S, Zheng B. Whole genome sequencing uncovers a novel IND-16 metallo-beta-lactamase from an extensively drug-resistant Chryseobacterium indologenes strain J31. Gut Pathog. 2016;8:47.

12. CLSI. Performance standards for antimicrobial susceptibility testing; $23 \mathrm{rd}$ informational supplement. Wayne: M100-S23: Clinical and Laboratory Standards Institute; 2013.

13. Zerbino DR, Birney E. Velvet: algorithms for de novo short read assembly using de Bruijn graphs. Genome Res. 2008;18:821-9.

14. Aziz RK, Bartels D, Best AA, DeJongh M, Disz T, Edwards RA, Formsma K, Gerdes S, Glass EM, Kubal M, et al. The RAST server: rapid annotations using subsystems technology. BMC Genom. 2008;9:75.

15. Natale DA, Shankavaram UT, Galperin MY, Wolf YI, Aravind L, Koonin EV. Towards understanding the first genome sequence of a crenarchaeon by genome annotation using clusters of orthologous groups of proteins (COGs). Genome Biol. 2000;1:RESEARCH0009.

16. Lowe TM, Eddy SR. tRNAscan-SE: a program for improved detection of transfer RNA genes in genomic sequence. Nucleic Acids Res. 1997;25:0955-64.

17. Lagesen $K$, Hallin P, Rødland EA, Stærfeldt H-H, Rognes T, Ussery DW. RNAmmer: consistent and rapid annotation of ribosomal RNA genes. Nucleic Acids Res. 2007;35:3100-8.

18. Arndt D, Grant JR, Marcu A, Sajed T, Pon A, Liang Y, Wishart DS. PHASTER: a better, faster version of the PHAST phage search tool. Nucleic Acids Res. 2016;44:W16-21.

19. Grissa I, Vergnaud G, Pourcel C. CRISPRFinder: a web tool to identify clustered regularly interspaced short palindromic repeats. Nucleic Acids Res. 2007;35:W52-7.

20. Carattoli A, Zankari E, Garcia-Fernandez A, Voldby Larsen M, Lund O, Villa L, Moller Aarestrup F, Hasman H. In silico detection and typing of plasmids using PlasmidFinder and plasmid multilocus sequence typing Antimicrob Agents Chemother. 2014;58:3895-903.

21. Siguier P, Varani A, Perochon J, Chandler M. Exploring bacterial insertion sequences with ISfinder: objectives, uses, and future developments. Methods Mol Biol. 2012;859:91-103.

22. Antipov D, Hartwick N, Shen M, Raiko M, Lapidus A, Pevzner PA. plasmidSPAdes: assembling plasmids from whole genome sequencing data. Bioinformatics. 2016;32:3380-7. 
23. Jia B, Raphenya AR, Alcock B, Waglechner N, Guo P, Tsang KK, Lago BA, Dave BM, Pereira S, Sharma AN, et al. CARD 2017: expansion and modelcentric curation of the comprehensive antibiotic resistance database. Nucleic Acids Res. 2017:45:D566-73.

24. Zankari E, Hasman H, Cosentino S, Vestergaard M, Rasmussen S, Lund O, Aarestrup FM, Larsen MV. Identification of acquired antimicrobial resistance genes. J Antimicrob Chemother. 2012;67:2640-4.

25. Chen L, Zheng D, Liu B, Yang J, Jin Q. VFDB 2016: hierarchical and refined dataset for big data analysis-10 years on. Nucleic Acids Res. 2016;44:D694-7.

26. Kleinheinz KA, Joensen KG, Larsen MV. Applying the ResFinder and VirulenceFinder web-services for easy identification of acquired antibiotic resistance and $E$. coli virulence genes in bacteriophage and prophage nucleotide sequences. Bacteriophage. 2014;4:e27943.

27. Carattoli A, Bertini A, Villa L, Falbo V, Hopkins KL, Threlfall EJ: Identification of plasmids by PCR-based replicon typing. J Microbiol Methods 2005;63:219-28.

28. Zheng B, Li A, Jiang X, Hu X, Yao J, Zhao L, Ji J, Ye M, Xiao Y, Li L. Genome sequencing and genomic characterization of a tigecycline-resistant Klebsiella pneumoniae strain isolated from the bile samples of a cholangiocarcinoma patient. Gut Pathog. 2014;6:40.

29. Schliep KP. phangorn: phylogenetic analysis in R. Bioinformatics. 2011;27:592-3.

30. Lam F, Lalansingh CM, Babaran HE, Wang Z, Prokopec SD, Fox NS, Boutros PC. VennDiagramWeb: a web application for the generation of highly customizable Venn and Euler diagrams. BMC Bioinform. 2016;17:401.

31. Rossi O, Baker KS, Phalipon A, Weill FX, Citiulo F, Sansonetti P, Gerke C, Thomson NR. Draft genomes of Shigella strains used by the STOPENTERICS consortium. Gut Pathog. 2015;7:14.

32. Zhang N, Lan R, Sun Q, Wang J, Wang Y, Zhang J, Yu D, Hu W, Hu S, Dai $\mathrm{H}$, et al. Genomic portrait of the evolution and epidemic spread of a recently emerged multidrug-resistant Shigella flexneri clone in China. J Clin Microbiol. 2014;52:1119-26.

33. Li J, Li B, Ni Y, Sun J. Molecular characterization of the extended-spectrum beta-lactamase (ESBL)-producing Shigella spp. in Shanghai. Eur J Clin Microbiol Infect Dis. 2015;34:447-51.

34. Xia S, Xu B, Huang L, Zhao JY, Ran L, Zhang J, Chen H, Pulsrikarn C, Pornruangwong S, Aarestrup FM, Hendriksen RS. Prevalence and characterization of human Shigella infections in Henan Province, China, in 2006. J Clin Microbiol. 2011;49:232-42.

35. Zhang CL, Liu QZ, Wang J, Chu X, Shen LM, Guo YY. Epidemic and virulence characteristic of Shigella spp. with extended-spectrum cephalosporin resistance in Xiaoshan District, Hangzhou, China. BMC Infect Dis. 2014;14:260.

36. Wang Y, Song C, Duan G, Zhu J, Yang H, Xi Y, Fan Q. Transposition of ISEcp1 modulates blaCTX-M-55-mediated Shigella flexneri resistance to cefalothin. Int J Antimicrob Agents. 2013;42:507-12.

37. Poirel L, Decousser JW, Nordmann P. Insertion sequence ISEcp1B is involved in expression and mobilization of a bla(CTX-M) beta-lactamase gene. Antimicrob Agents Chemother. 2003;47:2938-45.
38. Riccobono E, Di Pilato V, Villagran AL, Bartoloni A, Rossolini GM, Pallec chi L. Complete sequence of pV404, a novel Incl1 plasmid harbouring blaCTX-M-14 in an original genetic context. Int J Antimicrob Agents. 2014:44:374-6.

39. Valat C, Forest K, Billet M, Polizzi C, Saras E, Madec JY, Haenni M. Absence of co-localization between pathovar-associated virulence factors and extended-spectrum beta-lactamase (blaCTX-M) genes on a single plasmid. Vet Microbiol. 2016;192:163-6.

40. Bhagwat AA, Bhagwat M. Comparative analysis of transcriptional regulatory elements of glutamate-dependent acid-resistance systems of Shigella flexneri and Escherichia coli O157:H7. FEMS Microbiol Lett. 2004;234:139-47.

41. Edwards DJ, Streich FC Jr, Ronchi VP, Todaro DR, Haas AL. Convergent evolution in the assembly of polyubiquitin degradation signals by the Shigella flexneri IpaH9.8 ligase. J Biol Chem. 2014;289:34114-28.

42. Dogan B, Suzuki H, Herlekar D, Sartor RB, Campbell BJ, Roberts CL, Stewart K, Scherl EJ, Araz Y, Bitar PP, et al. Inflammation-associated adherentinvasive Escherichia coli are enriched in pathways for use of propanediol and iron and M-cell translocation. Inflamm Bowel Dis. 2014;20:1919-32.

43. Al-Hasani K, Navarro-Garcia F, Huerta J, Sakellaris H, Adler B. The immunogenic SigA enterotoxin of Shigella flexneri 2a binds to HEp-2 cells and induces fodrin redistribution in intoxicated epithelial cells. PLoS ONE. 2009; $4:$ e8223.

44. Wang F, Jiang Z, Li Y, He X, Zhao J, Yang X, Zhu L, Yin Z, Li X, Wang X, et al. Shigella flexneri T3SS effector IpaH4.5 modulates the host inflammatory response via interaction with NF-kappaB p65 protein. Cell Microbiol. 2013:15:474-85.

45. Tobe T, Nagai S, Okada N, Adler B, Yoshikawa M, Sasakawa C. Temperature-regulated expression of invasion genes in Shigella flexneri is controlled through the transcriptional activation of the virB gene on the large plasmid. Mol Microbiol. 1991;5:887-93.

46. Ruiz-Perez F, Wahid R, Faherty CS, Kolappaswamy K, Rodriguez L, Santiago A, Murphy E, Cross A, Sztein MB, Nataro JP. Serine protease autotransporters from Shigella flexneri and pathogenic Escherichia coli target a broad range of leukocyte glycoproteins. Proc Natl Acad Sci USA. 2011;108:12881-6.

47. Li P, Yang C, Wang J, Yi S, Li H, Wang Y, Qiu S, Song H. Draft genome sequence of a new Shigella flexneri subserotype, 4S BJ10610. Genome Announc. 2014;2:e00715.

48. Connor TR, Barker CR, Baker KS, Weill FX, Talukder KA, Smith AM, Baker S, Gouali M, Pham Thanh D, Jahan Azmi I, et al. Species-wide whole genome sequencing reveals historical global spread and recent local persistence in Shigella flexneri. Elife. 2015;4:e07335.

\section{Submit your next manuscript to BioMed Central and we will help you at every step:}

- We accept pre-submission inquiries

- Our selector tool helps you to find the most relevant journal

- We provide round the clock customer support

- Convenient online submission

- Thorough peer review

- Inclusion in PubMed and all major indexing services

- Maximum visibility for your research

Submit your manuscript at www.biomedcentral.com/submit 